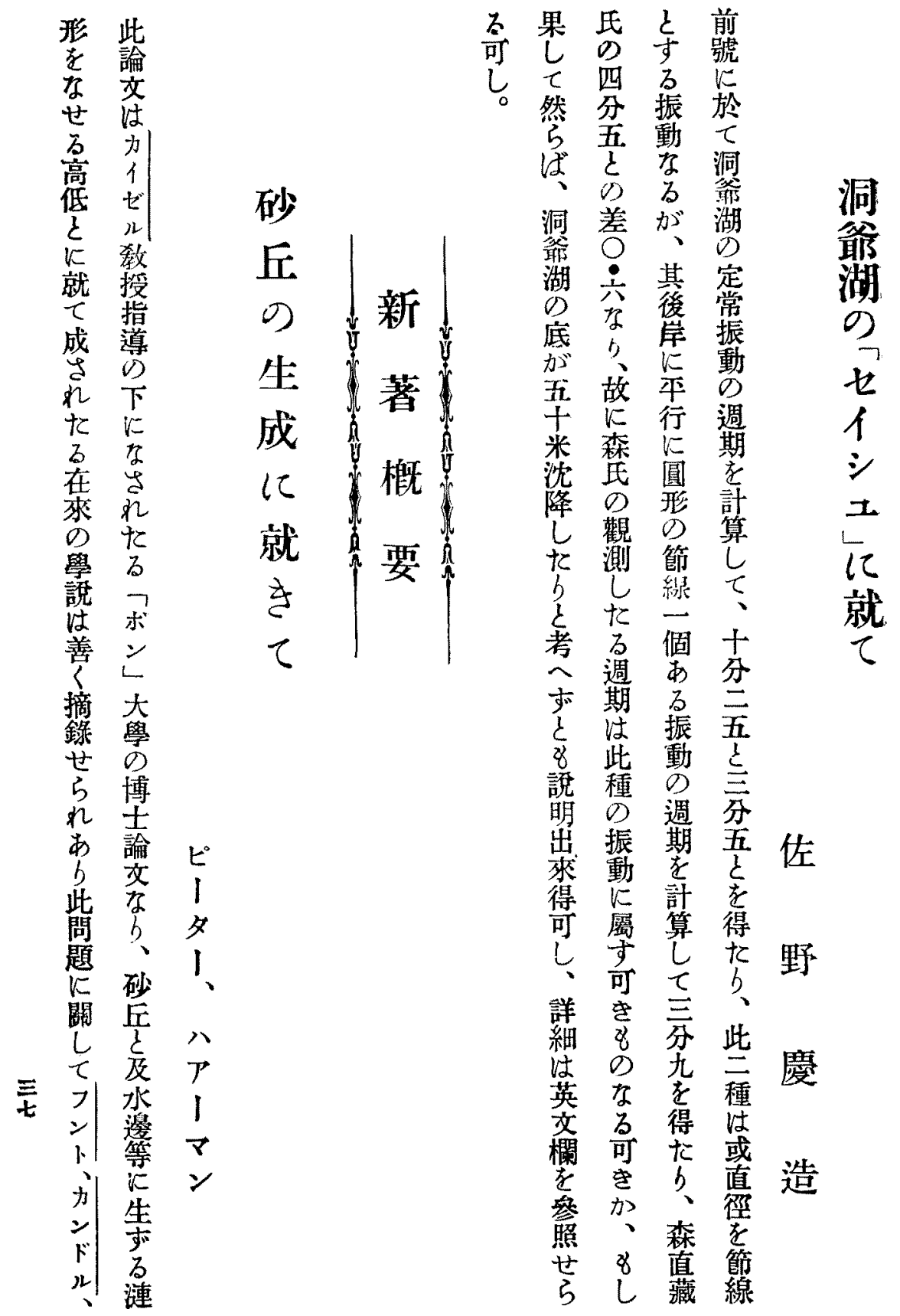




\section{ON THE SEICHES OF LAKE TÔYA.}

BY

\section{K. SANO.}

\section{Introduction.}

The scientific investigation of siches of Japanese lakes began in 1901 at the suggestion of Prof. Nagaoka. Prof. Honda(1) observed on the 18th and 19th of August, 1905, a period of 9.29 minutes in the seiches of I ake Tôya which is situated at $42^{\circ} 36^{\prime}$ $\mathrm{N}$. and $140^{\circ} 53^{\prime} \mathrm{E}$. in the island of Hokkaidô. The theoretical value of the period calculated by Prof. Honda and his colaborator from Merian's formula was 11.2 minutes. The discrepancy between the calculated and observed values is not great; but as the lake has an island in its centre, there would be better agreement between the theory and observation, if the presence of the island is taken into account in the calculation of the period. The author of the present paper had an opportunity to calculate theoretically the periods of the stiches of the same lake on the hypothesis different from those assumed by Prof. Honda, and found that they give closer agreement between the calculated and observed values.

In April, 1911, N. Mori (2) observed a period of 4.5 minutes in the same lake and suggested that its mean depth would have become about 50 metres deeper than in the time of Prof. Honda's observation. It seems to the author of this note that this corresponds to a certain mode of vibration of water in the lake and

(1) Journ. Coll. Sci., Imp. Univ. Tkôyô, vol. XVIII, p. 85,rgrt.

(2) Journ. Met. Soc. Jap., vol. XXX No 8, p. 73. 
can be explained without any change of depth.

\section{The Fundamentel Assumption to the theory.}

According to T. Kato's report(1) “On Lake Tôya," the lake is nearly circular in form and has an island also circular in form and almost concentric with the former. The radii of those circles are 5 and 1.2 kilometers respectively. In the paper already referred to Prof. Honda gives $9.58 \times 10^{3} \mathrm{~cm}$. for the mean depth of the lake.

We may, therefore, consider the water of the lake to be contained between two co-axial cylindrical surfaces whose common axis is vertical. The author assumes that the depth of the lake is uniform, because he can deal with the problem only by so doing. Let us denote the external and internal radii of the cylindrical surfaces by $a$ and $b$ respectively and the depth by $h$. By what has been stated we have

$$
a=5 \times 10^{5} \mathrm{~cm} ., b=1.2 \times 10^{5} \mathrm{~cm} ., h=9.58 \times 10^{3} \mathrm{~cm} .
$$

For the sake of simplicity let us also assume that water is a perfect incompressible fluid and that its motion is irrotational

\section{The Differential Equations.}

Let $u, v, w$ be the velocity components of water at the point $(x, y, z)$ at the instant $t$ respectively, and let $\mathrm{V}$ the velocity potential ; then

$$
u=-\frac{\partial \mathrm{V}}{\partial x}, \quad v=-\frac{\partial \mathrm{V}}{\partial y}, \quad w=-\frac{\partial \mathrm{V}}{\partial z} .
$$

As is well known the equation of continuity of the incompressible fluid is

(I) Rep. of the Earthqualse Investigation Committee, No. 65. 


$$
\frac{\partial u}{\partial x}+\frac{\partial v}{\partial y}+\frac{\partial w}{\partial z}=0,
$$

Combining equations (1) and $(\dot{2})$ together, we have

$$
\frac{\partial^{2} V}{\partial x^{2}}+\frac{\partial^{2} V}{\partial y^{2}}+\frac{\partial^{2} V}{\partial z^{2}}=0
$$

If $(r, \theta, z)$ are the cylindrical coordinates of the point $(x, y, z)$. equation (3) can be written

$$
\frac{\partial^{2} \mathrm{~V}}{\partial r^{2}}+\frac{1}{r} \frac{\partial \mathrm{V}}{\partial r}+\frac{1}{r^{2}} \frac{\partial^{2} \mathrm{~V}}{\partial \theta^{2}}+\frac{\partial^{2} \mathrm{~V}}{\partial z^{2}}=0
$$

Let the free surface when the fluid is at rest be the plane of $x y$, and let the axis of $z$ be taken vertically upwards. Then the boundary conditions to be satisfied by the fluid are as follows :-

(a) On the bottom of the lake, water can not flow vertically, so that

$$
\left(\frac{\partial V}{\partial z}\right)_{z=-h}=0
$$

(b) (i) On the external cylindrical surface which we consider as rigid, water can not flow in a radial direction, $i, e$.

$$
\left(\frac{\partial V}{\partial r}\right)_{r=a}=0
$$

(ii) the same consideration for the periphery of the island gives

$$
\left(\frac{\partial V}{\partial r}\right)_{r=b}=0
$$

(c) On the free surface we have the well-known approximate equation

$$
\left(\frac{\partial^{2} \mathrm{~V}}{\partial t^{2}}+g \frac{\partial \mathrm{V}}{\partial z}\right)_{x=0}=0
$$

when $g$ denotes the acceleration due to gravity and is equal to 


\section{$9.8 \times 10^{2} \mathrm{~cm} / \mathrm{sec}^{2}$}

We must now find particular solutions of (4) satisfying those boundary conditions. It is evident that the complete solution of (4) is impossible unless initial conditions are given.

Suppose that equation (4) is satisfied by $V=e^{p t i \pm n \theta i \pm k z} V_{1}$, where $p, n$ and $x$ are constants and $V_{1}$ is a function of $r$ only and $i$ is written for $\sqrt{-1}$. Then we have immediately

$$
\frac{\partial^{2} V_{1}}{\partial r^{2}}+\frac{1}{r} \frac{\partial V_{1}}{\partial r}+\left(x^{2}-\frac{n^{2}}{r^{2}}\right) V_{1}=0
$$

whose solution is

$$
\mathrm{V}_{1}=\mathrm{A}_{1} \mathrm{~J}_{n}(x \boldsymbol{r})+\mathrm{B}_{1} Y_{n}(x \boldsymbol{r})
$$

where $A_{1}$ and $B_{1}$ are constants of integration, and $J_{n}(\varkappa r)$ Bessel function of the $n$th order, and $\mathrm{Y}_{n}(\boldsymbol{x} \boldsymbol{r})$ any cylindrical function of the second kind of argument $u r$ and parameter $n$. Hence

$$
\mathrm{V}=e^{p \theta i \pm n t i \pm u_{1}}\left\{\mathrm{~A}_{1} \mathrm{~J}_{n}(u r)+\mathrm{B}_{1} \mathrm{Y}_{u}(u \boldsymbol{r})\right\}
$$

Equation (4) is also satisfied by multiplying the right-nand member by $e^{ \pm x h} / 2$, and therfore by

$$
\mathrm{V}=\frac{e}{2}^{p t i \pm n \theta i}\left\{e^{(i+h)}+e^{-u(z+n)}\right\}\left\{\mathrm{A}_{3} \mathrm{~J}_{n}(u r)+\mathrm{B}_{1} \mathrm{Y}_{n}(u r)\right\}
$$

i.e. by $\mathrm{V}=e^{p t i \pm n 0 i} \cosh x(z+h)\left\{\mathrm{A}_{1} \mathrm{~J}_{n}(\varkappa r)+\mathrm{B}_{1} \mathrm{Y}_{n}(\kappa r)\right\}$.

Since $\frac{\partial V}{\partial z}=\varkappa e^{p t i \pm n \theta i} \sin \kappa(z+h)\left\{\mathrm{A}_{1} \mathrm{~J}_{n}(\varkappa r)+\mathrm{B}_{1} Y_{n}(\kappa r)\right\}$,

it is evident that (9) satifies (5).

Now by (9)

$$
\frac{\partial V}{\partial r}=\varkappa e^{p t i \pm n \theta i} \cosh \varkappa(z+h)\left\{A_{1} J_{n}^{\prime}(\varkappa r)+B_{1} Y_{n}^{\prime}(\varkappa r)\right\} \text {, }
$$

so that (6) and (7) become respectively

$$
\mathrm{A}_{1} \mathrm{~J}_{n}{ }^{\prime}(\kappa a)+\mathrm{B}_{1} \mathrm{Y}_{n}^{\prime}(\kappa a)=0,
$$




$$
\mathrm{A}_{1} \mathrm{~J}_{n}{ }^{\prime}(\boldsymbol{x} b)+\mathrm{B}_{1} \mathrm{Y}_{n}^{\prime}(\boldsymbol{\kappa} b)=0 \text {. }
$$

Eliminating $A_{1}$, and $B_{1}$, from the last two equations we get

$$
\frac{J_{n}^{\prime}(\varkappa a)}{J_{n}^{\prime}(\kappa b)}=\frac{Y_{n}^{\prime}(\varkappa a)}{Y_{n}^{\prime}(x b)} \text {. }
$$

$\boldsymbol{x}$ is to be datermined from the transcendental equation (10).

From (9 it is easily seen that (4) is satisfied by

$$
\begin{aligned}
& \mathrm{V}=\cosh \varkappa(z+\mathrm{h})\left\{\frac{\left.\mathrm{J}_{n}{ }^{\prime} \varkappa r\right)}{\mathrm{J}_{n^{\prime}}{ }^{1}(\varkappa a)}-\frac{\mathrm{Y}_{n}(\varkappa r)}{\mathrm{Y}_{n}{ }^{\prime}(\varkappa a)}\right\}[\cos n \theta(\mathrm{A} \cos p t+\mathrm{B} \sin p t) \\
& +\sin n \theta(\mathrm{C} \cos p t+\mathrm{D} \sin p t)] \text {. }
\end{aligned}
$$

where $A, B, C, D$, are real constants. It is evident that (11) satisfies (5), and also (6) and (7) provided $x$ is so chosen that (10) is satisfied. Since $\mathrm{V}$ must have the same value when $\theta+2 \pi$ is put for $\theta, n$ is either $o$ or an integer.

\section{Calculation of the Roots of the equation $\frac{J_{n}{ }^{\prime}(\kappa(a)}{J_{n}{ }^{\prime}(\kappa b)}=\frac{Y_{n}{ }^{\prime}(\kappa a)}{Y_{n}{ }^{\prime}(\mu b)}$.}

If we put $x b=x, \frac{a}{b}=\rho$, then $\varkappa a=\rho x$, and (10) may be written

$$
\frac{d\left\lfloor J_{n}(\rho x)\right]}{d(\rho x)} \frac{d\left[Y_{n}(x)\right]}{d x}-\frac{\left.\left.d\lfloor\rfloor_{n} x^{\prime} x\right)\right]}{d x} \frac{d\left[\mathrm{Y}_{n}(\rho x)\right]}{d(\rho x)}=0
$$

Here it will be remarked that $\rho$ becomes 4.17 when the values of $a$ and $b$ given in $\$ 1$ are taken. If we write

$$
y=\frac{d\left[J_{n}(\rho x)\right]}{d(\rho x)} \frac{d\left[Y_{n}(x)\right]}{d x}-\frac{d\left[J_{n}(x)\right]}{d x} \frac{d\left[Y_{n}(\rho x)\right]}{d(\rho x)}
$$

then the equation for determining $x$ is

$$
y=0 \text {. }
$$

If $x_{n, s}$ is the sth positive root of (12), and $x_{n, s}$ the corresponding value of $\boldsymbol{\kappa}$, then $\boldsymbol{\kappa}_{n, y}$ can be determined by the relation

$$
x_{n, 8} b=x_{m, s} .
$$


The most general type of the motion of the liquid can be expressed by adding together the terms of the form given in (11) for all possible values of $n$ and $s$. To fix our idea let us take the simple case in which

$$
\mathrm{V}=\cosh \kappa_{n, s}(z+/ 2) \cos n \theta(\mathrm{A} \cos p t+\mathrm{B} \sin p t)\left\{\frac{J_{n}\left(\kappa_{n, n} r\right)}{J_{n}\left(\kappa_{n, 8} a\right)}-\frac{Y_{n}\left(\kappa_{n, s} r\right)}{Y_{n}^{\prime}\left(\kappa_{n, a} a\right)}\right\}
$$

Fig. I.

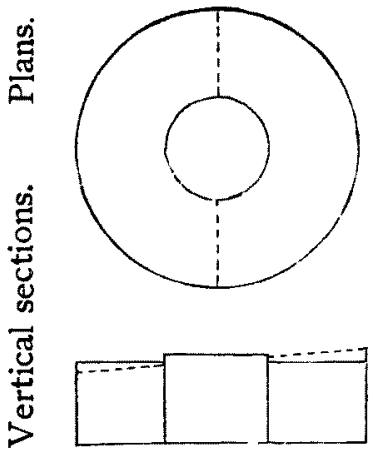

Fig. II.
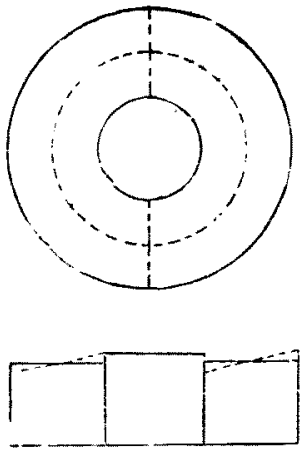

Fig. III.
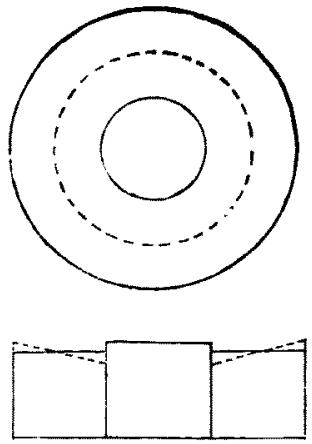

In the above figures the dotted lines in the vertical sections are intended to represent the suitable sections of the free surfaces. The locii of points on the free surfase at which the vertical component velocities of the liquid vanish, are denoted by broken lines. We shall call those lines apparent nodal lines.

1. Fig. I. corresponds to the case in which $n=1$ and $s=1$. We have here two nodal straight lines $\theta=\pi / 2$ and $\theta=3 \pi / 2$.

2. When $n=1$ and $s=2$, the vibration is the next harmonis of the preceding case; and as is given in Fig. II. we have an apparent nodal lines already mentioned.

* 3. In the case in which $n=0$ and $s=1$ we have only one apparent nodal line as is represented in Fig. III. 
The author has taken the case where $n=1$, and after transforming the exression of $y$ by the well-known equations

$$
\begin{aligned}
& \frac{d\left[\mathrm{Y}_{1}(x)\right]}{d x}=\mathrm{Y}_{0}(x)-\frac{\mathrm{Y}_{0}(x)}{x}, \quad \frac{d\left[\mathrm{~J}_{1}(x)\right]}{d x}=\mathrm{J}_{0}(x)-\frac{\mathrm{J}_{1}(x)}{x}, \\
& \frac{d\left[Y_{1}(\rho x)\right]}{d(\rho x)}=\mathrm{Y}_{0}(\rho x)-\frac{\mathrm{Y}_{1}(\rho x)}{\rho x}, \quad \frac{d\left[\mathrm{~J}_{1}(\rho x)\right]}{d(\rho x)}=\mathrm{J}_{0}(\rho a)-\frac{\mathrm{J}_{1}(\rho x)}{\rho x},
\end{aligned}
$$

he has calculated the first two roots of (12) by using the table given in Gray and Mathew's "Treatise on Bessel Functions" and by using Airy's tables ${ }^{(1)}$ of Neumann functions. The roots so calculated are

$$
x_{1,1}=0.40, \quad x_{1,2}=1.20 .
$$

\section{Periods of oscillation.}

From (8), the condition of the free surface, we can determine the period of oscillation. Differentiating (11) we get

$$
\begin{aligned}
& \frac{\partial^{2} \mathrm{~V}}{\partial t^{2}}=-p^{2} \cosh \boldsymbol{x}(z+h)\left\{\frac{\mathrm{J}_{n}(\varkappa r)}{\mathrm{J}_{n b}^{\prime}(\varkappa a)}-\frac{\mathrm{Y}_{n}(\varkappa r)}{\mathrm{Y}_{n}^{\prime}(\kappa a)}\right\}[\cos n \theta(\mathrm{A} \cos p t+\mathrm{B} \sin p t) \\
& +\sin n \theta(C \cos p t+D \sin p t)] \text {, } \\
& g \frac{\partial V}{\partial z}=g \kappa \sinh \kappa(z+h)\left\{\frac{\mathrm{J}_{n}(\kappa r)}{\mathrm{J}_{n}^{\prime}(\kappa a)}-\frac{\mathrm{Y}_{n}(\mu r)}{\mathrm{Y}_{n}^{\prime}(\kappa a)}\right\}[\cos n \theta(\mathrm{A}-\cos p t+\mathrm{B} \sin p t) \\
& +\sin n \theta(C \cos p t+D \sin p t)] \text {, }
\end{aligned}
$$

so that (8) becomes

$$
-p^{2} \cosh (x h)+\dot{x} g \sinh (x h)=0
$$

from which we obtain

$$
p^{2}=\varkappa g \tanh (x h)
$$

Let $p_{n, s}$ be the value of $p$ corresponding to $x_{n, s}$; then the equation just obtained can be written

(r) Phil. Mag., vol. XXII, p. 660, 19rr. 


$$
p_{n,}{ }^{2}=\kappa_{n, s} g \tanh \left(\varkappa_{n, s} h\right)
$$

so that the corresponding period is

$$
\mathrm{T}_{n, 8}=\frac{2 \pi}{p_{n, s}}=2 \pi \sqrt{\frac{\operatorname{coth}\left(\mu_{n, s} h\right)}{\mu_{n, 8} g}},
$$

Thus

$$
\mathrm{T}_{1,1}=2 \pi \sqrt{\frac{\operatorname{coth}\left(\varkappa_{1,2}, h\right)}{\kappa_{1,1} g}} .
$$

Since $\kappa_{1,1} h$ is small, we have approximately

$$
\operatorname{coth}\left(x_{1,1} g\right)=\frac{1}{x_{1,1} h}+\frac{x_{1,1} h}{3}
$$

whence we get

$$
\mathrm{T}_{1,1}=2 \pi \sqrt{\frac{1}{x_{1,1} g}\left(\frac{1}{\kappa_{1,1} h}+\frac{x_{11} h}{3}\right)}=2 \pi \sqrt{\frac{1}{\kappa_{1,1}{ }^{2} g h}+\frac{h}{3 g}} .
$$

Similarly we have

$$
\begin{aligned}
& \mathrm{T}_{1,2}=2 \pi \sqrt{\frac{1}{x_{1,2}{ }^{2} g h}+\frac{h}{3 g}}, \\
& \mathrm{~T}_{0,1}=2 \pi \sqrt{\frac{1}{x_{0,1}{ }^{2} g h}+\frac{h}{3 g}} .
\end{aligned}
$$

Putting (13) in (15) and (16), we find

$$
\begin{aligned}
& T_{1,1}=10.25 \text { minutes, } \\
& T_{1,2}=3.5 \text { minutes. }
\end{aligned}
$$

Thus the period of oscillation of the type $n=1, s=1$ is 10.25 minutes, that is, greater than the observed value 9.29 minutes by about $10 \%$.

\section{The case in which $n=0$.}

The author had an opportunity to calculate the case in which $n=0$ and $s=1$, i.e. the case of Fig. III. 
In this case, by virtue of the well-known equations

$$
\frac{d\left[\mathrm{~J}_{0}(x)\right]}{d x}=-\mathrm{J}_{1}(x), \frac{d\left[\mathrm{Y}_{\mathrm{n}}(x)\right]}{d x}=-\mathrm{Y}_{0}(x), \& \mathrm{c} .,
$$

we have

$$
y=\mathrm{J}_{1}(\rho) \mathrm{Y}_{1}(x)-\mathrm{J}_{1}(x) \mathrm{Y}_{1}(\rho x) .
$$

By using the tables already referred to the author obtains

$$
x_{0,1}=u_{0,1} b=1.06 \text {, }
$$

so that $\mathrm{T}_{0,1}=3 \cdot 9$ minutes.

As already stated period observed by Mori was $4 . \overline{0}$ minutes. The difference between the calculated and observed values are not great so that there is a possibility that the observed case can be explained as the oscillation of the above type; but as the simultaneous observations at different places on the lake were not given by the observer, we can not be sure whether the observed oscillation corresponds really to the case in which $n=0$ and $s=1$, or not.

In conclusion the author wishes to express his heartiest thanks to Prof. S. Sano for his kind guidance. 